\title{
VAC.03 - Polymeric nanoparticles as immunoadjuvant for oral immunization
}

Juliana de Souza Rebouças ${ }^{1 *}$; Fabio Rocha Formiga ${ }^{2}$; Juan Manuel Irache ${ }^{3}$; Carlos Gamazo ${ }^{3}$.

1UPE;

2Fiocruz - Fundação Oswaldo Cruz;

3Universidad de Navarra (Espanha).

Introduction: Peanut allergy is reaching epidemic proportions, with important socioeconomic consequences. Besides, peanuts are the most common cause of food related anaphylaxis and death. In recent years, different strategies for allergen immunotherapy have been sought. In this context, a promising strategy is the use of polymeric nanoparticles (NP) as antigen delivery systems. NP act increasing the immunogenicity of encapsulated allergens but also may present immunomodulatory activity in order to elicit a balanced immune response.

Objective: The aim of this work was to evaluate the potential application of polymeric NP as immunoadjuvants for oral immunization against peanut allergy.

Methodology: Female C57BL/6J mice (Harlan, Spain), 8 weeks old (20 \pm 1 g), were randomly divided into six groups ( $n=5$ per group). Animals were orally immunised with a single dose of the following formulations: (i) lyophilized raw PE-entrapped nanoparticles (NP-RaPELF); (ii) lyophilized roasted PE-entrapped nanoparticles (NP- RoPE-LF); (iii) spray-dried raw PE-entrapped nanoparticles (NP-RaPE-SD); or (iv) spray- dried roasted PE-entrapped nanoparticles (NP-RoPE-SD). Non-loaded NP (NP-LF and NP- SD) and free peanut proteins (raw and roasted) were administered as controls. Animals were euthanized five weeks after initial immunization. The presence of PE-specific antibodies was determined in sera (IgG1 and IgG2a) by indirect ELISA. The presence of cytokines (IL-5 and IFN-gamma) was determined by ELISA. Animal Experimentation was approved by University of Navarra (protocol number 048/09).

Results: The adjuvant effect of NP formulations was demonstrated by a stronger and balanced $\mathrm{T}_{\mathrm{H}} 1$ (IgG2a) and $\mathrm{T}_{\mathrm{H}} 2$ (IgG1) specific antibody response. All animals immunized with the proteins encapsulated into NP showed higher levels of IgG1 and IgG2a antibodies than the groups that received the proteins in their free forms (raw or roasted, ro PE). In addition, as for allergenicity, animals immunized with spray-dried NP showed lower specific IgE compared to the groups that received the proteins in their free forms (raw and roasted). Furthermore, spray-dried-NP elicited a significant decrease in $\mathrm{T}_{\mathrm{H}} 2$ cytokines (IL-4, IL-5 and IL-6) accompanied by an enhancement of both regulatory (IL-10) and $\mathrm{T}_{\mathrm{H}} 1$ cytokine (INF- $\gamma$ ) secretion, with low IgE induction. In order to evaluate the potential of the NP formulations to induce mucosal responses, we quantified specific-IgA produced in faecal content after oral immunization. All NP formulations (L or SD) were able to elicit higher levels of intestinal IgA compared to free PE, especially for NP loaded with raw peanut proteins (NP-Raw-L vs. raw PE; NP-Raw-SD vs. Raw PE).

Conclusion: Oral immunization with poly(anhydride) NP, particularly spray-dried formulations, led to a robust pro- $\mathrm{T}_{\mathrm{H}} 1$ immune response, characterized by a high $\mathrm{T}_{\mathrm{H}} 1 / \mathrm{T}_{\mathrm{H}} 2$ ratio with low IgE response. Thus, poly(anhydride) nanoparticles represent a promising tool for oral immunotherapy against peanut allergy.

Keywords: Polymeric nanoparticles; Immunoadjuvants; Allergy 